\title{
A NOVEL NON-DIFFRACTIVE RECONSTRUCTION METHOD FOR DIGITAL HOLOGRAPHIC MICROSCOPY
}

\author{
Michael Liebling ${ }^{\dagger}$, Thierry Blu ${ }^{\dagger}$, Etienne Cuche ${ }^{\dagger}$, \\ Pierre Marquet ${ }^{\ddagger}$, Christian Depeursinge ${ }^{\dagger}$, Michael Unser ${ }^{\dagger}$ \\ †Institut d'Imagerie et Optique Appliquée, Swiss Federal Institute of Technology, Lausanne \\ ${ }^{\ddagger}$ Institut de Physiologie, Université de Lausanne, Switzerland
}

\begin{abstract}
We present a new method for reconstructing digitally recorded off-axis Fresnel holograms. Currently-used reconstruction methods are based on the simulation and propagation of a reference wave that is diffracted by the hologram. This procedure introduces a twin-image and a zero-order term which are inherent to the diffraction phenomenon. These terms perturb the reconstruction and limit the field of view. Our new approach splits the reconstruction process into two parts. First, we recover the amplitude and the phase in the camera plane from the measured hologram intensity. Our algorithm is based on the hypothesis of a slowly varying object wave which interferes with a more rapidly varying reference wave. In a second step, we propagate this complex wave to refocus it using the Fresnel transform. We therefore avoid the presence of the twin-image and zeroorder interference terms. This new approach is flexible and can be adapted easily to complicated experimental setups. We demonstrate its feasibility in the case of digital holographic microscopy and present results for the imaging of living neurons.
\end{abstract}

\section{INTRODUCTION}

Digital holography $[1,2]$ is an imaging method in which a hologram [3] is recorded with a CCD-camera and reconstructed numerically. Digital holography has been applied successfully to microscopy and is particularly suited for the imaging of living biological samples as it is truly non-invasive. The reconstructed phase provides direct information on the morphology of the cells; i.e. the height of the specimen. Most remarkable is the ability of the technique to detect sub-wavelength changes in the morphology of the studied samples [4].

The hologram results from the interference between the complex wave reflected or transmitted by the object to be imaged and a complex reference plane wave. The object's three-dimensional information is thus encoded in one single, two-dimensional, real-valued image. This allows for a

This work is part of the joint project in biomedical engineering of HUG/UNIL/EPFL/UNIGE/HCV: Microdiag high acquisition rate and makes the technique highly suitable for tracking biological processes.

The most widely used reconstruction technique consists in simulating the propagation of a reference wave that is diffracted by the hologram, imitating the physical phenomenon. The main pitfall of this approach — and most common source of criticism made to digital off-axis holography is that the reconstructed image is corrupted by interference terms, the zero-order and twin-image, which substantially restrict the visual field. While several techniques have been proposed to remove these terms [5, 6], they still remain a determining factor that limits the quality of the reconstructed image.

Here, we propose a new holographic reconstruction method that essentially solves this problem. In a first step, we apply a new algorithm that retrieves the complex wave in the CCD plane from the real-valued measures. Once we have recovered this information, we (back)propagate the wave (which does neither contain the zero-order nor the twinimage term) to restore a focused image using the Fresnel transform.

\section{DIGITAL HOLOGRAPHIC MICROSCOPY}

\subsection{Experimental setup}

The experimental setup for digital holographic microscopy is similar to the one described in [4]; it is schematically depicted in Figure 1. The specimen is illuminated by a plane wave and the transmitted light is collected by a microscope objective that produces a wave front called object wave. The CCD camera records the interference of this object wave with a reference plane wave in an off-axis geometry. This latter terminology refers to the fact that the reference wave impinges on the camera with a slight angle. The CCD is at a distance $d$ from the image plane. This implies that the complex wave that we are attempting to recover in the camera plane is out of focus and will require a propagation step to yield the desired image of the specimen. This will be accomplished by using a numerical version of the Fresnel transform [7], which approximates the diffraction integral [8]. 


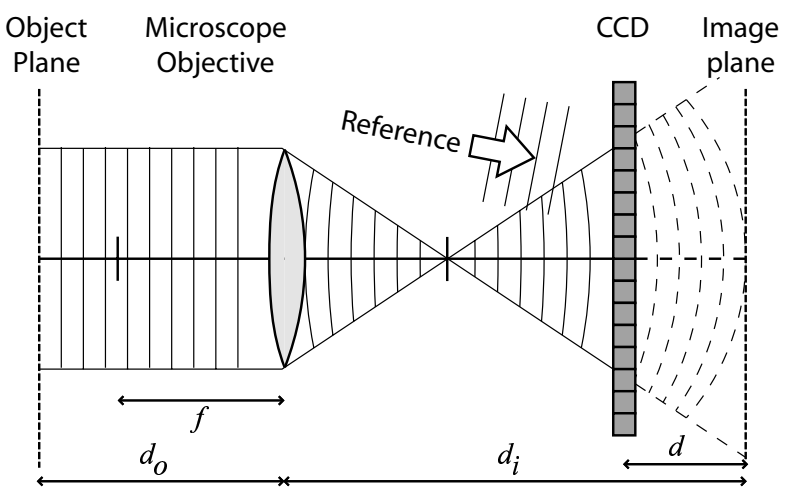

Fig. 1. Schematic of the holographic microscope. An object is illuminated by a plane wave. The microscope objective has a focal length $f$. The CCD is at a distance $d$ from the object's image. The off-axis reference wave interferes with the object wave and produces the hologram in the CCD plane.

Furthermore, the presence of the microscope objective implies that the object wave in the CCD plane $\Psi(\vec{x})$ is multiplied by a quadratic-phase exponential $[8,4]$ :

$$
\Psi(\vec{x}) \exp \left(i \frac{1}{D}\|\vec{x}\|^{2}\right)
$$

where $D$ is a function of $d, d_{i}$ and the focal length $f$.

\subsection{Interference}

The intensity (or hologram) measured by the CCD camera, $I(\vec{x}) \in \mathbb{R}_{+}$is the result of the interference between the object wave and a reference (usually plane) wave $R_{p}$ :

$$
I(\vec{x})=\left|\Psi(\vec{x}) \exp \left(i \frac{1}{D}\|\vec{x}\|^{2}\right)+R_{p}(\vec{x})\right|^{2} .
$$

We rewrite this expression to get:

$$
I(\vec{x})=|\Psi(\vec{x})+R(\vec{x})|^{2}
$$

where we consider the more general expression for the reference wave $R(\vec{x})=A(\vec{x}) \exp (i \theta(\vec{x}))\left(A(\vec{x}) \in \mathbb{R}_{+}, \theta(\vec{x}) \in\right.$ $\mathbb{R})$. Note that the measure $I(\vec{x})$ is real while the wave to recover $\Psi(\vec{x})$ is complex. In other words, at each measured location $\vec{x}$, we have only one equation for two unknowns (the real and imaginary part of $\Psi(\vec{x})$ ), assuming that the reference wave $R(\vec{x})$ is known.

\section{PHASE RETRIEVAL}

The key idea of our algorithm is that we consider $I(\vec{x})$ and $\theta(\vec{x})$ to be the only quantities that vary rapidly in space. Also, as $\theta(\vec{x})$ can easily be modeled, we consider it to be known. Conversely, we assume that the unknown quantities $\Psi(\vec{x})$ and $A(\vec{x})$ vary slowly (or a least less rapidly than the reference wave) so that we can regard them as constant within the neighborhood of a given point of interest $\vec{x}$. With this hypothesis, we can determine the phase and amplitude of $\Psi(\vec{x})$ together with $A(\vec{x})$ by solving the following set of $M$ non-linear equations for each location $\vec{x}$ :

$$
I\left(\vec{x}+\vec{x}_{m}\right)=\left|\Psi(\vec{x})+A(\vec{x}) \exp \left(i \theta\left(\vec{x}+\vec{x}_{m}\right)\right)\right|^{2}
$$

where the $\vec{x}+\vec{x}_{m}(m=1, \ldots, M)$ are the locations of the $M$ pixels within the considered neighborhood of $\vec{x}$. Simplifying the notation, we have:

$$
\begin{aligned}
I_{m} & =\left|\Psi+A \exp \left(i \theta_{m}\right)\right|^{2} \\
& =|\Psi|^{2}+A^{2}+2 \Re\left(R_{m}^{*} \Psi\right) .
\end{aligned}
$$

Next, we introduce the auxiliary variables $\phi=\frac{\Psi}{A}, U=$ $A^{2}+\frac{1}{A^{2}}|\phi|^{2}, V_{m}=\exp \left(i \theta_{m}\right)$ and we solve (5) in the leastsquares sense, which is equivalent to determine:

$$
\arg \min _{U, \phi} \sum_{m}\left|I_{m}-U-2 \Re\left(V_{m} \phi\right)\right|^{2} .
$$

Consequently, $U$ and $\phi$, must be the solutions to the following set of normal equations:

$$
\left\{\begin{array}{l}
\sum_{m} I_{m}-U-2 \Re\left(V_{m} \phi\right)=0 \\
\sum_{m} V_{m}\left(I_{m}-U-2 \Re\left(V_{m} \phi\right)\right)=0 \\
\sum_{m} V_{m}^{*}\left(I_{m}-U-2 \Re\left(V_{m} \phi\right)\right)=0
\end{array}\right.
$$

obtained by differentiating the expression in (6) with respect to $U$ and $\phi$. Rearranging the terms, we get:

$$
\left\{\begin{array}{l}
\frac{1}{M} \sum_{m} I_{m}=U+2 \Re\left(\phi \frac{1}{M} \sum_{m} V_{m}\right) \\
\frac{1}{M} \sum_{m} V_{m} I_{m}=U \frac{1}{M} \sum_{m} V_{m}+\left(\frac{1}{M} \sum_{m} V_{m}^{2}\right) \phi+\phi^{*} \\
\frac{1}{M} \sum_{m} V_{m}^{*} I_{m}=U \frac{1}{M} \sum_{m} V_{m}^{*}+\phi+\left(\frac{1}{M} \sum_{m} V_{m}^{* 2}\right) \phi^{*} .
\end{array}\right.
$$

Finally, by setting $v=\frac{1}{M} \sum_{m} V_{m}$ and $w=\frac{1}{M} \sum_{m} V_{m}^{2}$, we end up with a linear set of equations to solve:

$$
\left(\begin{array}{lll}
1 & v & v^{*} \\
v & w & 1 \\
v^{*} & 1 & w^{*}
\end{array}\right)\left(\begin{array}{l}
U \\
\phi \\
\phi^{*}
\end{array}\right)=\left(\begin{array}{l}
\frac{1}{M} \sum_{m} I_{m} \\
\frac{1}{M} \sum_{m} V_{m} I_{m} \\
\frac{1}{M} \sum_{m} V_{m}^{*} I_{m}
\end{array}\right) .
$$

The only remaining point is to model the reference wave's phase $\theta(\vec{x})$ accurately. To do so, we used a parametric model with four parameter, $D_{x}, D_{y}, k_{x}, k_{y}$ :

$$
\theta(\vec{x})=\frac{1}{D_{x}} x^{2}+\frac{1}{D_{y}} y^{2}+k_{x} x+k_{y} y .
$$

The parameters $D_{x}$ and $D_{y}$ depend on the quadratic phase introduced by the lens while $k_{x}$ and $k_{y}$ depend on the reference wave's incidence angle. In general, these quantities cannot be obtained experimentally with the required level of accuracy. However, in the case of a transmission setup for holographic microscopy, the phase distribution over a flat background area can be assumed to be constant and the reconstruction parameters adjusted accordingly. 


\section{RESULTS AND DISCUSSION}

We have applied this reconstruction technique to the imaging of living neurons in the context of a functional morphology experiment. The neurons were grown in a perfusion chamber (cf. Figure 2). We use our system to track the

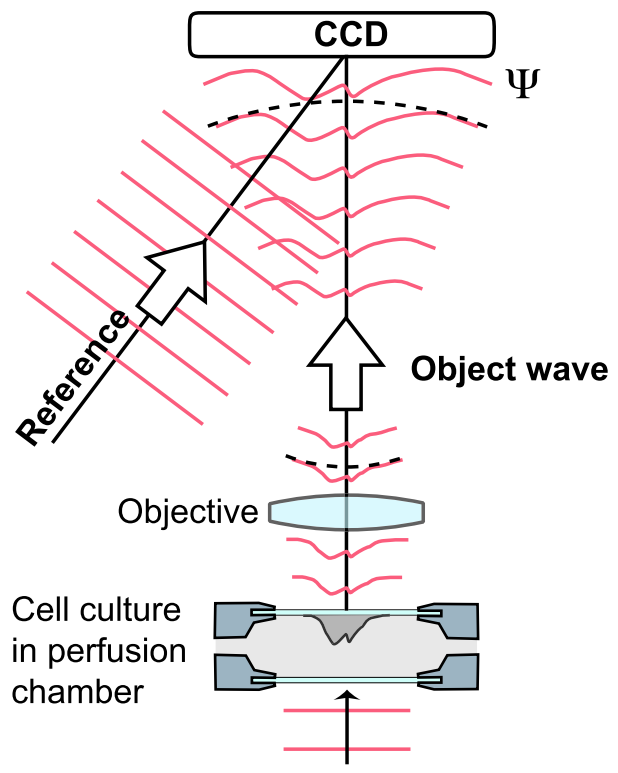

Fig. 2. Schematic of the holographic microscope for the imaging of living neurons

change of the cell morphology in response to a variation of the perfusion liquid's concentration. This time phenomenon can be observed at video rate since our system only requires one data acquisition per reconstructed image. One frame of the intensity measured on the CCD camera is shown in Figure 3. The circular fringes are the result of the interference of the quadratic-phase exponential induced by the objective lens and the reference plane wave. It corresponds to the expression of $I(\vec{x})$ in equation (3) with the reference wave's phase $\theta(\vec{x})$ given by (10).

The reconstruction using the standard technique described in [4] is shown in Figure 4. In this approach, the quadratic-phase exponential induced by the objective is compensated numerically by multiplying the diffracted wave by a function of the form (10). The zero-order is the square portion that masks the center of the image. The image itself is on the upper right while the twin-image is located in the lower left. Only the image is in focus.

In Figure 5, we show the phase in the CCD plane (i.e. $\arg \Psi$, following the notation of the previous sections) that was recovered with our new algorithm. The complex values at each pixel were estimated using a sliding window of size $5 \times 5$, i.e. $M=25$. The bodies of the neurons are already recognizable (white blobs) but the image is blurred since the CCD camera is not in the image plane. The recovered

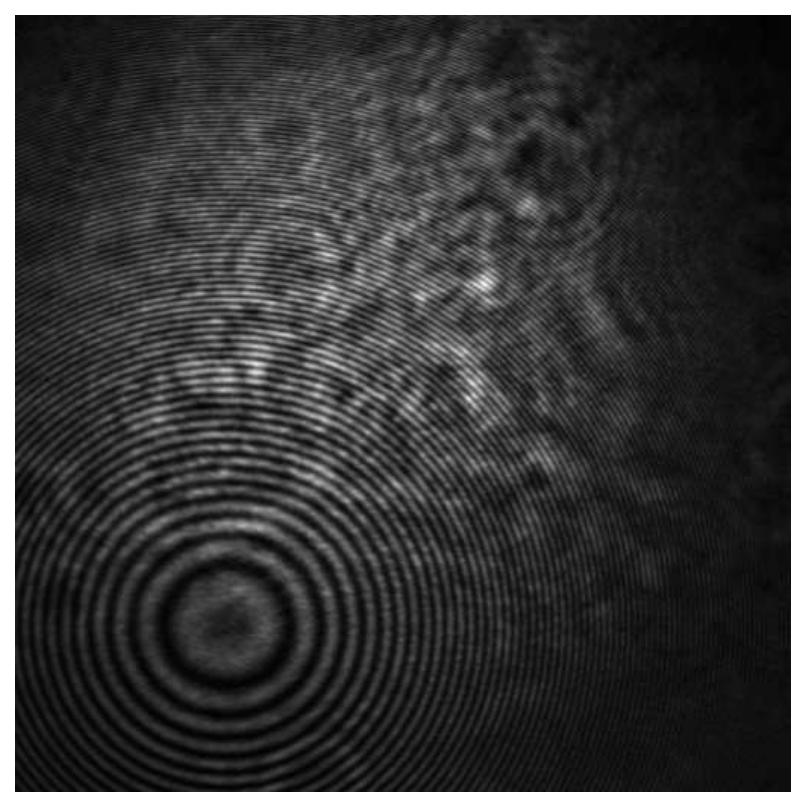

Fig. 3. Measured hologram. $512 \times 512$ pixels, the sampling step is $10 \mu \mathrm{m}$.

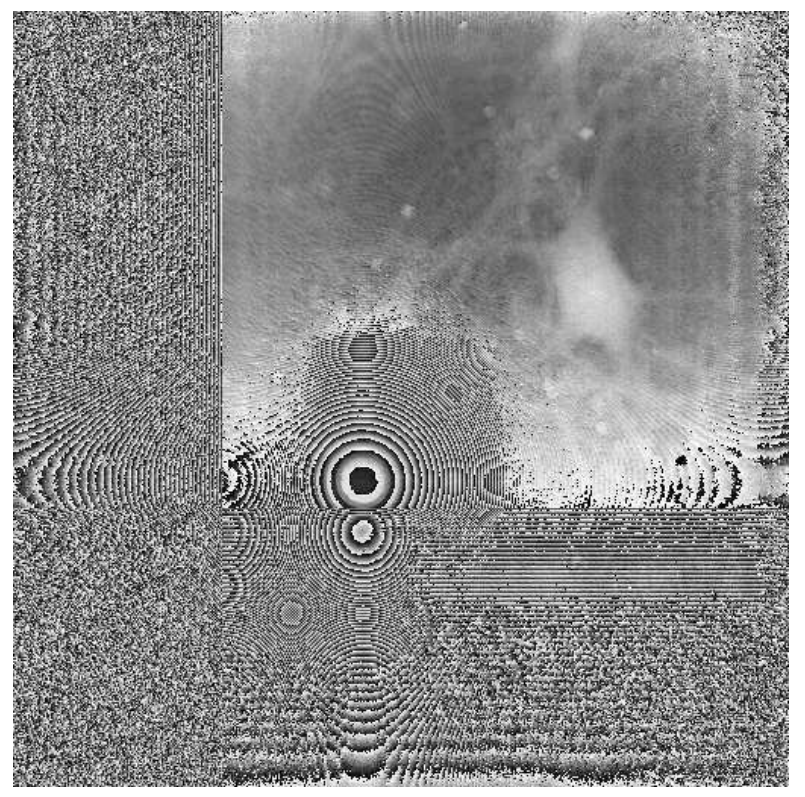

Fig. 4. Reconstructed phase with the method described by Cuche et al. [4] $(512 \times 512$ pixels $)$.

wave front in the CCD plane is then propagated to the image plane at the proper distance $d$; the final reconstructed image is shown in Figure 6. The reconstruction distance was set to $d=10.1 \mathrm{~cm}$. Remarkably, if we compare this reconstruction with the one in Figure 4, we see that it is clearer and not perturbed by the zero-order. A second neuron that was previously hidden is now visible in the field of view. Note 


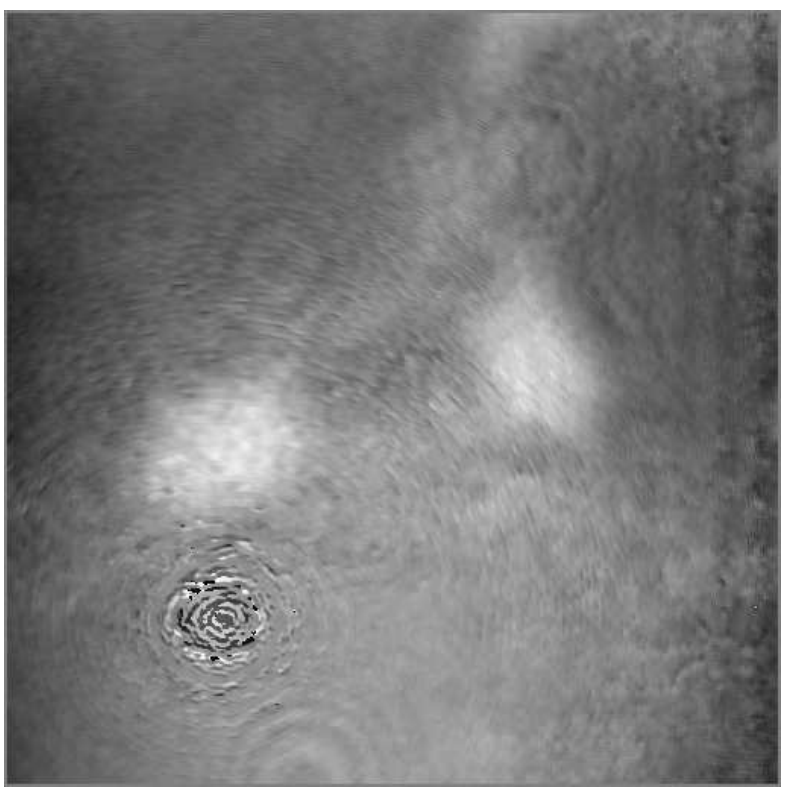

Fig. 5. Phase in the CCD plane $(512 \times 512$ pixels $)$.

that the image is reconstructed at a finer resolution than in the previous approach.

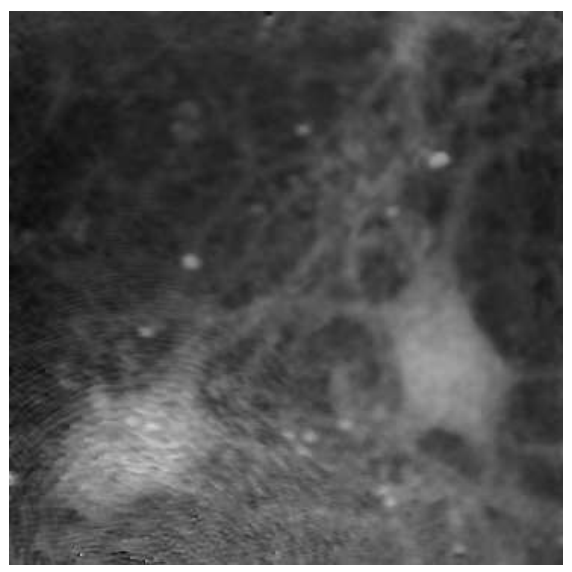

Fig. 6. Reconstructed phase (detail, $366 \times 366$ pixels).

\section{CONCLUSION}

We have demonstrated the feasibility of an alternative hologram reconstruction technique which treats the phase retrieval problem and the propagation problem separately. The results obtained so far are very promising. The new approach has several advantages. First, it yields reconstructions that are not affected by the zero-order and twin-image interference terms. Second, it is more flexible because it clearly decouples the various problems. Consequently, it should remain applicable over a broader range of experimental conditions while keeping the advantages of the offaxis geometry which requires a single acquisition per image. With the reconstruction method that we are proposing, there is no waste of data and this should contribute to making digital holographic microscopy an even more useful modality. Unlike conventional microscopy, it can provide quantitative phase informations with remarkable precision. It can also operate at video rates which makes it very suitable for the study of dynamic biological phenomena.

\section{REFERENCES}

[1] J. W. Goodman and R. W. Lawrence, "Digital image formation from electronically detected holograms," Applied Physics Letters, vol. 11, no. 3, pp. 77-79, August 1967.

[2] L. P. Yaroslavskii and N. S. Merzlyakov, Methods of Digital Holography, Consultants Bureau, New York, 1980.

[3] D. Gabor, "A new microscopic principle," Nature, vol. 161, no. 4098, pp. 777-778, May 1948.

[4] E. Cuche, P. Marquet, and Ch. Depeursinge, "Simultaneous amplitude-contrast and quantitative phasecontrast microscopy by numerical reconstruction of Fresnel off-axis holograms," Applied Optics, vol. 38, no. 34, pp. 6994-7001, December 1999.

[5] E. Cuche, P. Marquet, and Ch. Depeursinge, "Spatial filtering for zero-order and twin-image elimination in digital off-axis holography," Applied Optics, vol. 39, no. 23, pp. 4070-4075, August 2000.

[6] T. M. Kreis and W. P. O. Jüptner, "Suppression of the dc term in digital holography," Optical Engineering, vol. 36, no. 8, pp. 2357-60, August 1997.

[7] M. Liebling, T. Blu, and M. Unser, "Fresnelets-A new wavelet basis for digital holography," in Proceedings of the SPIE Conference on Mathematical Imaging: Wavelet Applications in Signal and Image Processing IX, San Diego CA, USA, July 29-August 1 2001, vol. 4478, pp. 347-352.

[8] J. W. Goodman, Introduction to Fourier Optics (Second ed.), McGraw-Hill Companies, Inc., New York, 1996. 